\title{
High-power Broadband Organic THz Generator
}

SUBJECT AREAS:

NONLINEAR OPTICS

TERAHERTZ OPTICS

Received

11 July 2013

Accepted

22 October 2013

Published

13 November 2013

Correspondence and requests for materials should be addressed to

F.R. (rotermun@ajou. ac.kr) or O-P.K.

(opilkwon@ajou.ac.kr)

* These authors contributed equally to this work.
Jae-Hyeok Jeong ${ }^{1 *}$, Bong-Joo Kang ${ }^{2 *}$, Ji-Soo Kim' ', Mojca Jazbinsek', Seung-Heon Lee', Seung-Chul Lee', In-Hyung Baek ${ }^{2}$, Hoseop Yun ${ }^{4}$, Jongtaek Kim ${ }^{5,6}$, Yoon Sup Lee ${ }^{5}$, Jae-Hyeok Lee', Jae-Ho Kim?', Fabian Rotermund ${ }^{2} \&$ O-Pil Kwon'

'Department of Molecular Science and Technology, Ajou University, Suwon 443-749, Korea, ${ }^{2}$ Department of Physics \& Division of Energy Systems Research, Ajou University, Suwon 443-749, Korea, ${ }^{3}$ Rainbow Photonics AG, Farbhofstrasse 21, CH-8048 Zurich, Switzerland, ${ }^{4}$ Department of Chemistry \& Division of Energy Systems Research, Ajou University, Suwon 443-749, Korea,

${ }^{5}$ Department of Chemistry, Korea Advanced Institute of Science and Technology (KAIST), Daejeon 305-701, Korea, ${ }^{6}$ Department of Basic Science, Korea Air Force Academy, Cheongiu 363-849, Korea.

The high-power broadband terahertz $(\mathrm{THz})$ generator is an essential tool for a wide range of $\mathrm{THz}$ applications. Here, we present a novel highly efficient electro-optic quinolinium single crystal for $\mathrm{THz}$ wave generation. For obtaining intense and broadband $\mathrm{THz}$ waves by optical-to- $\mathrm{THz}$ frequency conversion, a quinolinium crystal was developed to fulfill all the requirements, which are in general extremely difficult to maintain simultaneously in a single medium, such as a large macroscopic electro-optic response and excellent crystal characteristics including a large crystal size with desired facets, good environmental stability, high optical quality, wide transparency range, and controllable crystal thickness. Compared to the benchmark inorganic and organic crystals, the new quinolinium crystal possesses excellent crystal properties and $\mathrm{THz}$ generation characteristics with broader $\mathrm{THz}$ spectral coverage and higher $\mathrm{THz}$ conversion efficiency at the technologically important pump wavelength of $800 \mathrm{~nm}$. Therefore, the quinolinium crystal offers great potential for efficient and gap-free broadband $\mathrm{THz}$ wave generation.

- erahertz (THz) spectroscopy and imaging systems in the frequency range from 0.1 to $30 \mathrm{THz}$ have recently attracted considerable attention for numerous applications ${ }^{1-5}$. To bridge the so-called "THz gap" , the development of an efficient and broadband $\mathrm{THz}$ generator is a key challenge in modern THz technologies $^{1,3,7,8}$. In addition to well-known photoconductive antennas and inorganic nonlinear optical crystals often used as $\mathrm{THz}$ emitters ${ }^{9-11}$, organic nonlinear electro-optic crystals exhibiting larger macroscopic nonlinearity and low dielectric constant have attracted increasing interests for efficient and broadband $\mathrm{THz}$ wave generation ${ }^{8,12-15}$. In recent years, two different novel approaches-wave-front-tilted excitation in prism-type $\mathrm{LiNbO}_{3}$ and non-phasematched rectification in laser-induced gas/air plasma with amplified Ti:sapphire laser systems-have been successfully demonstrated to produce very intense broadband $\mathrm{THz}$ waves with electric fields up to the $\mathrm{MV} / \mathrm{cm}$ or higher and energies in the microjoule range ${ }^{16-19}$. However, these methods, generally require high pump energies, precise alignments and complex experimental schemes. Although the use of organic crystals cannot directly compete with these methods, efficient $\mathrm{THz}$ waves generated by optical-to- $\mathrm{THz}$ frequency conversion can be easily realized with moderate energies in relatively simple traveling-wave geometries ${ }^{8,12-15,20-22}$.

To generate efficient broadband $\mathrm{THz}$ waves in organic crystals, a large macroscopic electro-optic response and optimal crystal characteristics, including a large crystal size with desired facets, high optical quality, good environmental stability, and large transparency range are require ${ }^{23}$. In addition, the possibility of controlling the crystal thickness and thereby of optimizing the group velocity-matched interaction length between the optical and $\mathrm{THz}$ waves is very crucial for obtaining high conversion efficiency and material transparency in optical and $\mathrm{THz}$ range ${ }^{13,15,24}$. During the past four decades, many electro-optic crystals have been intensively investigated ${ }^{25-31}$; however, only few crystals with large electro-optic response of over $50 \mathrm{pm} / \mathrm{V}$ have been reported ${ }^{23,32-37}$. Moreover, meeting all the requirements with simultaneous realization of optimal crystal characteristics for efficient $\mathrm{THz}$ generation is extremely difficult in a medium.

In this paper, we report a novel organic electro-optic crystal, HMQ-TMS (2-(4-hydroxy-3-methoxystyryl)-1methylquinolinium 2,4,6-trimethylbenzenesulfonate), applicable for efficient $\mathrm{THz}$ wave generation with pumping at $800 \mathrm{~nm}$, which corresponds to the operation wavelength of the technologically important and widespread femtosecond Ti:sapphire laser sources. For optical-to-THz frequency conversion process, HMQ-TMS crystal 


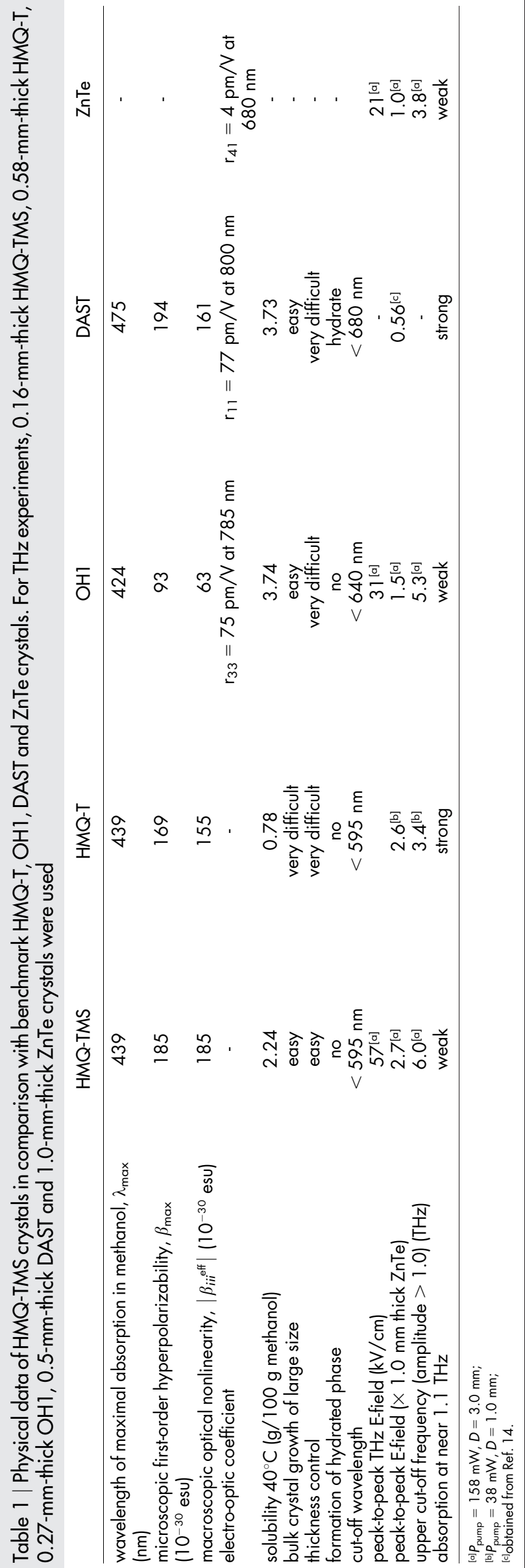

fulfills all the requirements such as a large electro-optic response and optimal crystal characteristics, including a large crystal size with desired facets, high environmental and thermal stabilities, high optical quality, extremely broad transparency range, and the possibility to control the crystal thickness. Moreover, HMQ-TMS crystals with various thicknesses (in the range of $0.05 \mathrm{~mm}$ up to few millimeters) that can be controlled by employing a simple cleaving method, can be fabricated to achieve minimal group velocity mismatch between the optical and $\mathrm{THz}$ waves at a desired optical pump wavelength. Although perfect phase matching condition cannot be realized at the pump wavelength of $800 \mathrm{~nm}$, a 0.16-mm-thick HMQTMS crystal can produce a much broader $\mathrm{THz}$ spectrum and about 2.7-times-higher $\mathrm{THz}$ generation efficiency than the widely used inorganic ZnTe crystal ( $1 \mathrm{~mm}$ thick) that operates under the nearly group velocity-matched condition at this pump wavelength. Furthermore, when compared with other organic benchmark ionic and nonionic crystals, which are generally incapable of pumping at $800 \mathrm{~nm}$, HMQ-TMS crystals exhibit excellent crystal properties and $\mathrm{THz}$ generation characteristics with broader $\mathrm{THz}$ spectral coverage and show the highest $\mathrm{THz}$ conversion efficiency with $800-\mathrm{nm}$ pumping.

\section{Results}

Design of high-efficiency electro-optic crystal. For an effective optical-to-THz frequency conversion via either optical rectification (OR) or difference frequency generation $(\mathrm{DFG})^{8,12-15,20-22}$, a large macroscopic electro-optic response is the primary requirement for electro-optic crystals. State-of-the-art organic crystals, such as stilbazolium DAST ( $N, N$-dimethylamino- $N^{\prime}$-methylstilbazolium 4methylbenzenesulfonate $)^{32,33}$, DSTMS ( $N, N$-dimethylamino- $N^{\prime}$-methylstilbazolium 2,4,6-trimethylbenzenesulfonate) ${ }^{13,34}$, DAPSH $(N, N$ -dimethylamino- $N^{\prime}$-phenyl-4-stilbazolium hexafluorophosphate $)^{35,36}$, quinolinium HMQ-T (2-(4-hydroxy-3-methoxystyryl)-1-methylquinolinium 4-methylbenzenesulfonate) and its derivatives ${ }^{37}$, and phenolic polyene OH1 (2-(3-(4-hydroxystyryl)-5,5-dimethylcyclohex2-enylidene)malononitrile $)^{23}$ exhibit electro-optic responses that are at least one order of magnitude larger than that of the inorganic standard ZnTe crystal $^{23,33,38}$ (see Table 1).

In order to design new organic crystals with large electro-optic responses, we chose an asymmetric core HMQ (2-(4-hydroxy-3methoxystyryl)-1-methylquinolinium) cation, since it shows a strong tendency to form acentric crystal structures based on Coulomb interactions ${ }^{37,39-41}$ and since it exhibits a large optical transparency range ${ }^{37}$. Further, 2,4,6-trimethylbenzenesulfonate (TMS) anion was chosen as the counter anion. In organic stilbazolium crystals, an increase in the number of methyl groups on benzenesulfonate anion resulted in improved crystal growth characteristics and lower absorption in the $\mathrm{THz}$ spectral range ${ }^{13,34}$. The chemical structure of the newly designed quinolinium HMQ-TMS is shown in Figure 1a.

The HMQ-TMS compound was synthesized by metathesis of 2(4-hydroxy-3-methoxystyryl)-1-methylquinolinium iodide with a silver precursor (see Supplementary Information (SI)). The results of differential scanning calorimetry (DSC) and thermal gravimetric analysis (TGA) measurements (see Figure S1 in SI) show that the HMQ-TMS crystal displayed high environmental and thermal stability with a melting temperature $\mathrm{T}_{\mathrm{m}}$ of $274^{\circ} \mathrm{C}$, and no thermal phase transition and weight loss was observed at temperature $T_{i}$ of $280^{\circ} \mathrm{C}$. Moreover, the results of single-crystal X-ray diffraction analysis indicate that HMQ-TMS single crystals grown in a methanol solution by rapid cooling method exhibited the monoclinic $P n$ space group with point group symmetry $m$, based on Coulomb interaction and strong hydrogen bonds. The crystal packing diagram of HMQTMS crystal is shown in Figure $1 \mathrm{~b}$.

For the evaluation of electro-optic response of HMQ-TMS crystal, the macroscopic optical nonlinearities were calculated by quantum mechanical calculations using finite field (FF) density functional 
(a)<smiles>COC1CC(/C=C/C2CCC3CCCCC3N2C)CCC1O</smiles>

(b)

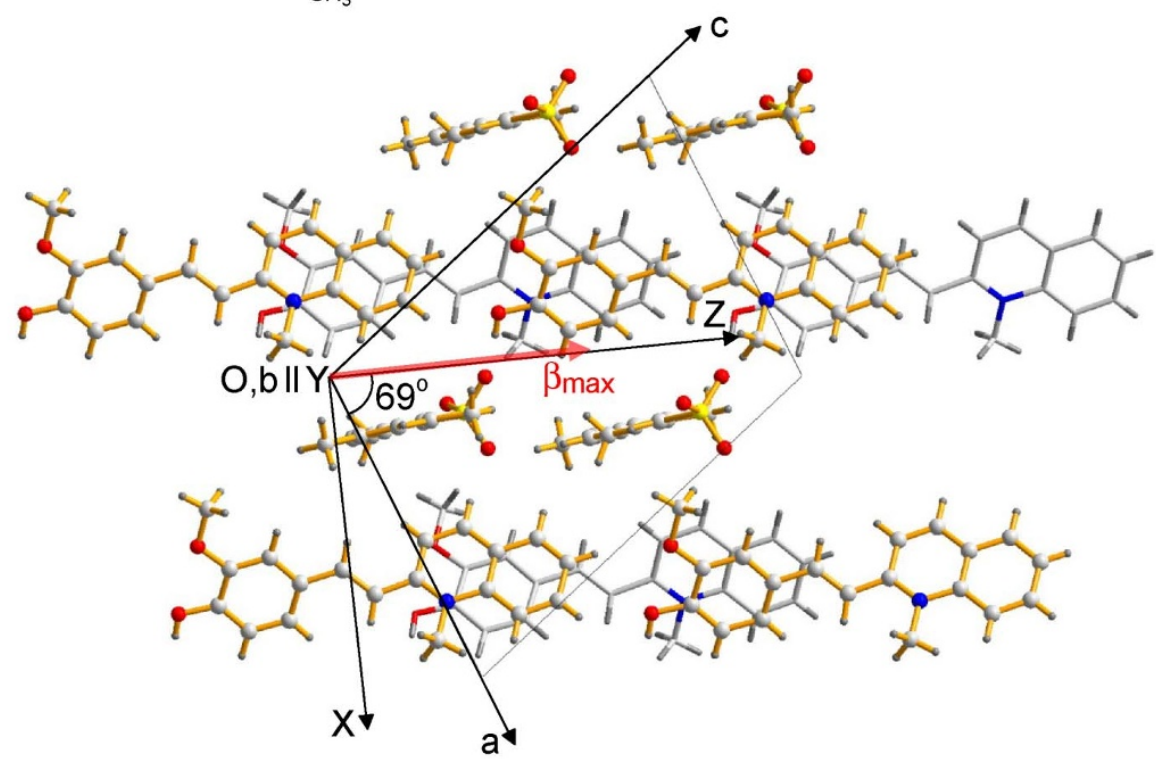

Figure $1 \mid$ Chemical structure and molecular orientation in the crystalline state. (a) Chemical structure of HMQ-TMS. (b) Crystal packing diagram of HMQ-TMS crystals projected along the $b$-axis. The molecular-ordering angle of $\beta_{\max }$ with respect to the main charge transfer axis $Z$ (polar axis) of the crystal is very small $\left(\theta_{p}=1.1^{\circ}\right)$, which results in a practically perfect order parameter $\cos ^{3} \theta_{p}=1.0$.

theory (DFT) with B3LYP/6-311 + $\mathrm{G}^{* 42,43}$, by considering the molecular conformation and orientation of HMQ cations as in the crystal structure (see SI $)^{44}$. The major calculation results are summarized in Table 1, where the results from the well-known organic and inorganic crystals are also listed for comparison ${ }^{23,37,45}$. The microscopic optical nonlinearity in the HMQ-TMS crystals, that is the first-order hyperpolarizability along the main charge-transfer direction $\beta_{\max }$ of the HMQ cation, is $185 \times 10^{-30}$ esu. Although HMQ-TMS shows the maximum absorption at much shorter wavelength $\left(\lambda_{\max }=439 \mathrm{~nm}\right)$ in methanol solution than that of DAST $\left(475 \mathrm{~nm}\right.$ ) (see Figure S2 in SI), $\beta_{\max }$ of the former was comparable to that of the DAST cation $\left(194 \times 10^{-30} \mathrm{esu}\right)$ in the crystalline state ${ }^{37,45}$.

In addition, the HMQ-TMS crystal exhibited optimal molecular packing structure for maximizing the electro-optic response. As shown in Figure 1b, HMQ nonlinear molecules were aligned perfectly parallel, resulting in the highest possible order parameter of $\cos ^{3} \theta_{p}=1.0$. Due to the large microscopic optical nonlinearity and optimal molecular packing in the crystalline state, the HMQ-TMS crystal exhibited a larger diagonal component of the macroscopic electro-optic response with effective hyperpolarizability tensor ( $\beta_{333}^{\text {eff }}$ $=185 \times 10^{-30} \mathrm{esu}$ ), as compared to OH1, DAST, and HMQ-T crystals (see Table 1). The demonstrated large electro-optic response in HMQ-TMS has therefore made it a promising candidate for $\mathrm{THz}$ wave applications.

Optimal crystal characteristics for THz generation. Bulk HMQTMS crystals were grown by a slow-cooling method in solution. Large amounts of HMQ-TMS compound were synthesized by a condensation reaction (see SI). Figure 2 a shows the solubility curve of HMQ-TMS crystals in methanol; a high solubility of HMQ-TMS crystalline powder is seen for example, $2.24 \mathrm{~g} / 100 \mathrm{~g}$ methanol at $40^{\circ} \mathrm{C}$, which is about 3.5 times higher than that of HMQ-T crystals $^{37}$. HMQ-T crystals exhibited a high $\mathrm{THz}$ generation efficiency at the near-infrared (NIR) optical wavelength, but only relatively small crystal sizes with a width of less than $1.5 \mathrm{~mm}$ could be grown due to low solubility ${ }^{37}$. This small crystal size substantially limits their applications in the generation of high $\mathrm{THz}$ electric fields. In contrast to HMQ-T, HMQ-TMS showed very good crystal growth characteristics, which was also due to the high solubility and the considerably large decrease in solubility as a function of temperature. Figure $2 \mathrm{~b}$ shows a photograph of a HMQ-TMS crystal grown by slow cooling method with spontaneous nucleation at a saturation temperature of $40^{\circ} \mathrm{C}$ in methanol. A bulk crystal grown in a seeded solution is shown in Figure S3 in SI. The grown HMQ-TMS crystals showed a relatively large aperture and thickness with sizes up to $18 \times$ $15 \times 8 \mathrm{~mm}^{3}$.

The environmental stability of crystals plays an important role in their applications in practical devices. In general, salt-type crystals can be dissolved in water and easily degraded by humidity. In addition, the formation of centrosymmetric hydrate phase is one of the most serious issues. For example, a stilbazolium DAST crystal can be dissolved in water and form a centrosymmetric hydrated phase when it is grown from water-contaminated solvents ${ }^{45,46}$. When HMQ-TMS crystals grown in water solvent mixture (water/methanol $=1: 9 \mathrm{v} / \mathrm{v}$ ), their thermodiagrams obtained from DSC and powder X-ray diffraction patterns are practically identical as the ones for HMQ-TMS crystals grown in methanol only (see SI). Furthermore, the solubility of HMQ-TMS in water has been examined and measured after stirring for 30 hours and it was found to be $0.07 \mathrm{~g} / 100 \mathrm{~g}$ water, which is 1.5 times lower than that of DAST $(0.11 \mathrm{~g} / 100 \mathrm{~g}$ water $)$ at $20^{\circ} \mathrm{C}$. 
(a)

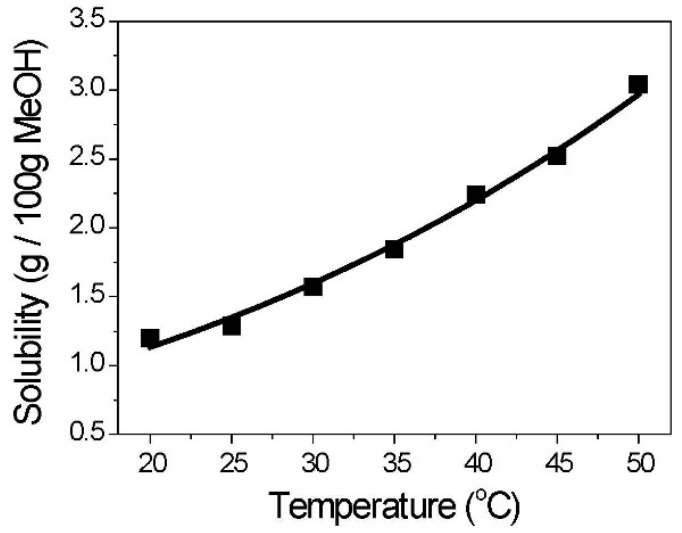

(b)

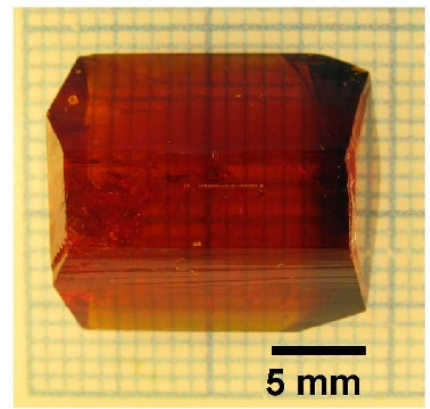

(c)
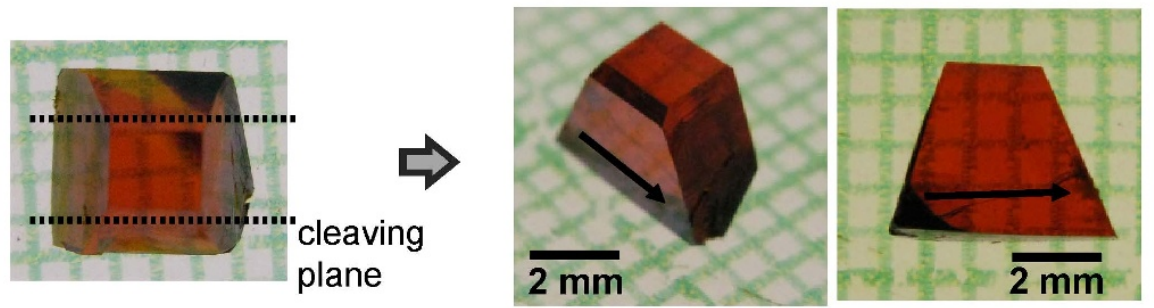

(d)

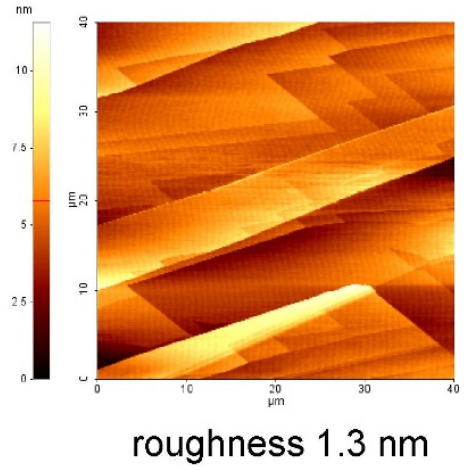

(e)

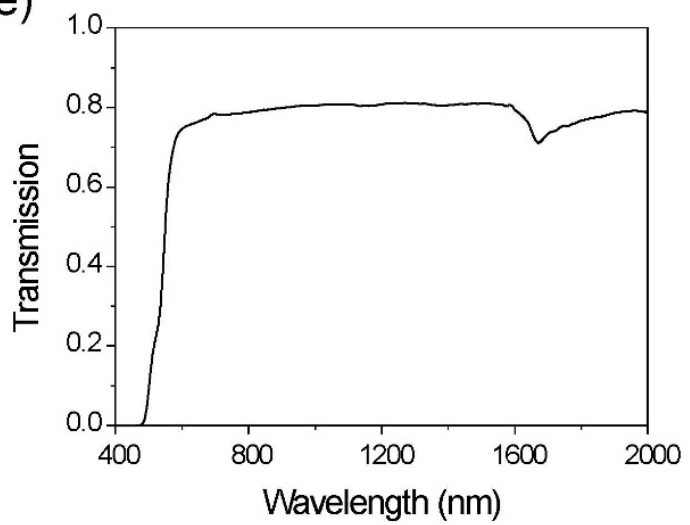

Figure $2 \mid$ Optimal crystal characteristics for THz generation. (a) Solubility curve of HMQ-TMS in methanol. (b) Photograph of a large HMQTMS crystal grown by slow cooling method with spontaneous nucleation at a saturation temperature of $40^{\circ} \mathrm{C}$ in methanol. (c) Photographs of HMQTMS crystals after cleaving along the crystallographic ac-plane by a diamond knife. The polar axis, which is presented by the solid vector, is in the ac-plane. (d) AFM image $\left(40 \times 40 \mu \mathrm{m}^{2}\right)$ of the cleaved surface. (e) Transmission spectrum of a cleaved HMQ-TMS sample with a thickness of 0.14 mm without polishing, using unpolarized light.

Therefore, HMQ-TMS crystals demonstrated better environmental stability and humidity resistance than DAST crystals did.

For all the optical experiments including that for $\mathrm{THz}$ generation, parallel sample surfaces with desired crystallographic facets are essential $^{47,48}$. To maximize the $\mathrm{THz}$ generation efficiency, the polar axis should be particularly along the surface plane of the crystal and parallel to the polarization of the incident pump beam. Since asgrown HMQ-TMS crystals do not have a perfect hexahedron shape as shown in Figure 2b, their direct application is rather limited. However, HMQ-TMS crystals having parallel surfaces and desired facets can be easily prepared by a simple method of cleaving along the crystallographic ac-plane using a diamond knife, as shown in Figure $2 c$. The quality of the cleaved surfaces was examined by atomic force microscopy (AFM). As depicted in Figure 2d (more in Figure S5 in SI), the cleaved surface was very flat and smooth with a surface roughness of less than $1.3 \mathrm{~nm}$ within an area of $40 \times$ $40 \mu^{2}$; this level of smoothness is very difficult to achieve by using mechanical or chemical polishing methods ${ }^{49}$. The cleaved HMQ-TMS crystals exhibited good optical quality and are stable performance without undesired cleavage or cracking during all the experiments. Figure $2 \mathrm{e}$ shows the transmission spectrum of a cleaved HMQ-TMS crystal with a thickness of $0.14 \mathrm{~mm}$ without additional polishing process. A non-polarized light, incident normal to the cleavage ac-plane, was used in this measurement, and no scattering centers were observed. As we expected, HMQ-TMS crystal exhibited a wide optical transparency range with a relatively low cut-off wavelength $\lambda_{\text {cut-off }}$ of $<595 \mathrm{~nm}$, which is lower than that of the benchmark OH1 and DAST crystals and similar to that of HMQ-T crystals (see Table 1).

Generally, in the optical-to- $\mathrm{THz}$ frequency conversion process, the thickness of nonlinear electro-optic crystals determines the dispersion and interaction lengths of optical and $\mathrm{THz}$ beams, and the thickness therefore strongly influences both conversion efficiency and spectral bandwidth of the generated $\mathrm{THz}$ waves ${ }^{13,15}$. The optimal crystal thickness is typically in the range of $0.05 \mathrm{~mm}$ to several millimeters, depending on material properties, the wavelength of optical pump beam, and its pulse width ${ }^{13,15,20}$. Therefore, the possibility of easy control of the crystal thickness, which allows optimization of 
group velocity matching between the optical and $\mathrm{THz}$ waves, is very important for efficient broadband $\mathrm{THz}$ wave generation. Although benchmark organic crystals exhibit large nonlinear and electro-optic responses, the fabrication of these samples with the desired area and optimal thickness is a very difficult issue. OH1, DAST and DSTMS might be grown in large areas over $1 \mathrm{~cm}^{2}$, but a large thickness is simultaneously obtained according to their natural growth morpho$\log y^{13,34,50,51}$. When the desired thickness is realized, either only smallarea crystals will be available after growth or very sophisticated cutting and polishing procedures are required. Moreover, such crystals are especially appropriate for phase matching at longer pump wavelengths in the $1.2-1.6 \mu \mathrm{m}$ range, where their optimal thickness may reach the millimeter level. However, for the NIR region below $1 \mu \mathrm{m}$, which is the typical operation wavelength of widespread and technologically important Ti:sapphire lasers, the optimum thickness is very thin due to increased dispersion: typically $<0.1 \mathrm{~mm}$ for DAST and DSTMS crystals and $<0.2 \mathrm{~mm}$ for OH1 crystals ${ }^{13,15,20}$. Such a low thickness is extremely difficult to produce and crystal damage can be easily caused during the polishing process due to mechanical stress ${ }^{47,50,51}$. HMQ-T crystals exhibited similar thickness limitations; moreover, as-grown HMQ-T crystals showed a relatively small area with sides of length $<1.5 \mathrm{~mm}$, as discussed above $\mathrm{e}^{37}$. (a)

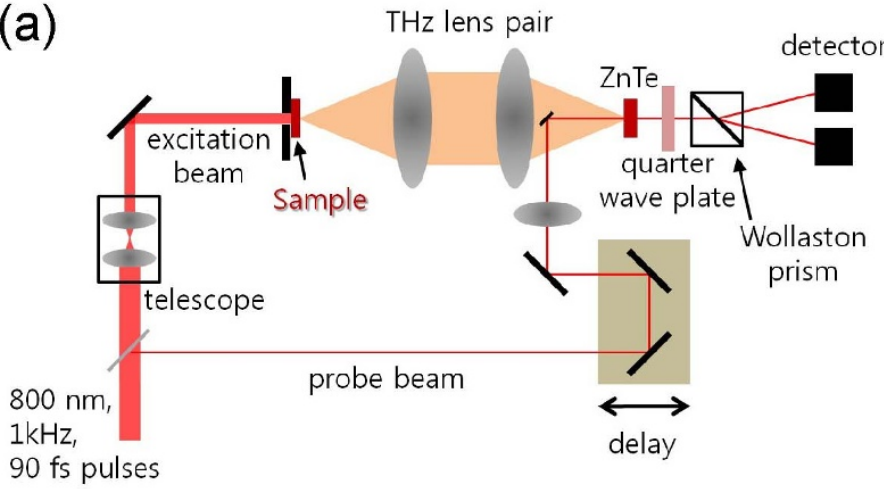

(c)

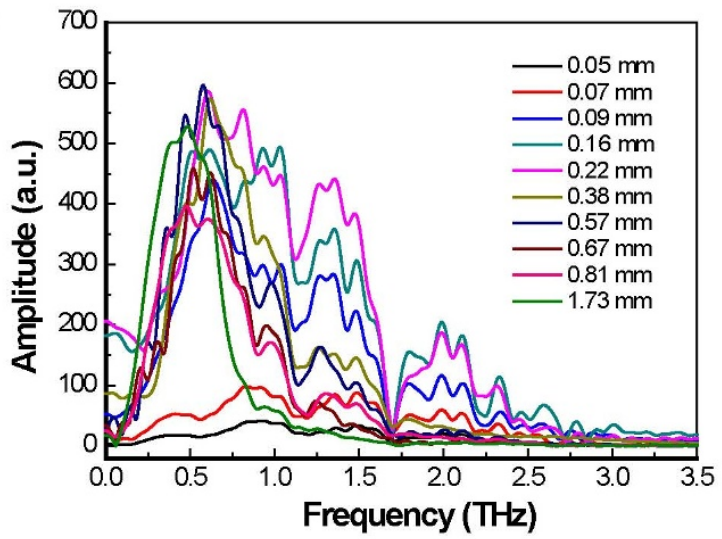

(e)

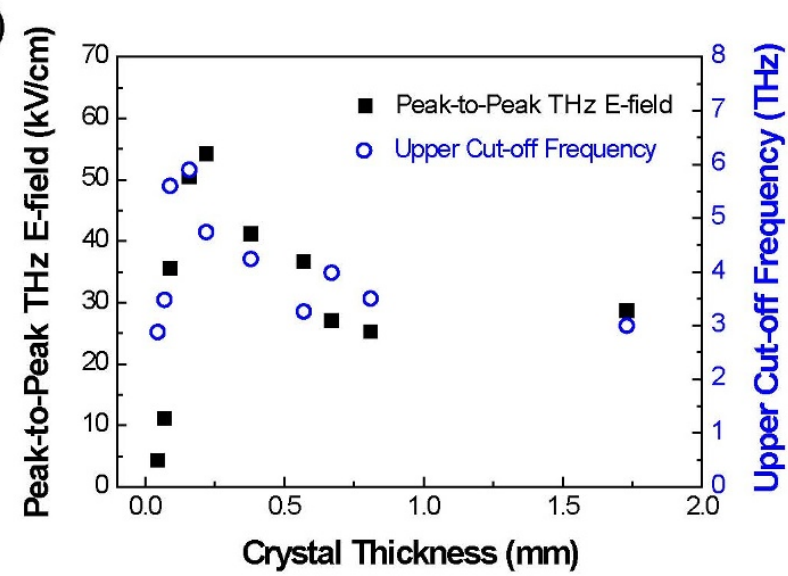

(b)

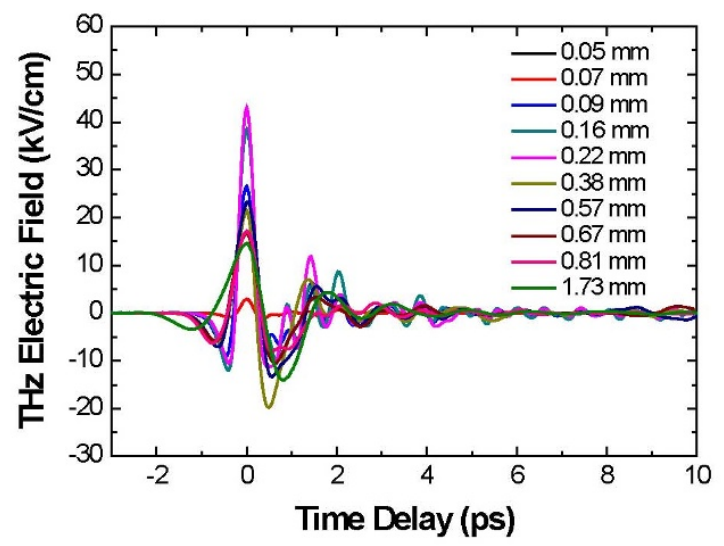

(d)

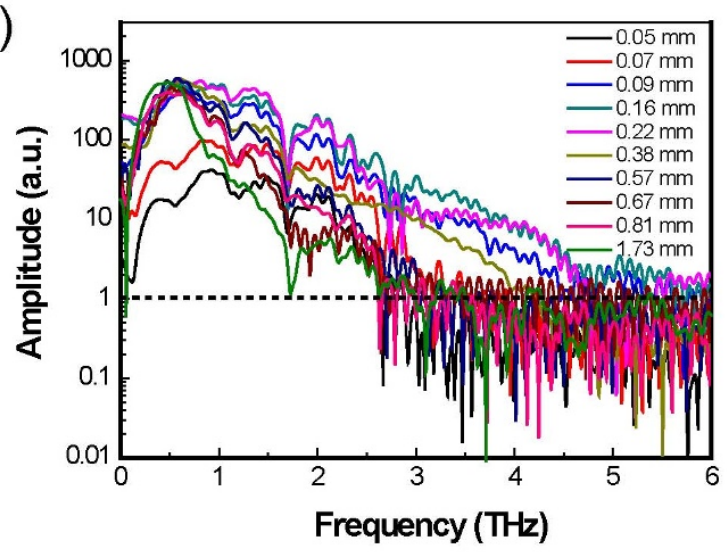

(f)

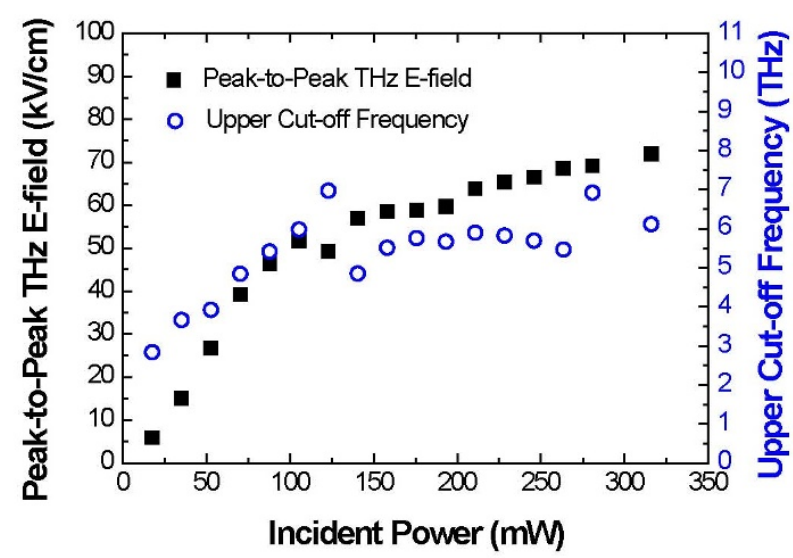

Figure 3 THz wave generation with the best group velocity-matched conditions. (a) THz wave generation setup. THz wave generation in HMQ-TMS crystals of different thicknesses $\left(P_{\text {pump }}=62 \mathrm{~mW}, D=1.7 \mathrm{~mm}\right)$ : (b) time-domain $E_{\mathrm{THz}}(t)$ traces, $(\mathrm{c})$ the corresponding frequency spectra of $E_{\mathrm{THz}}(v)$, (d) the frequency spectra of $E_{\mathrm{THz}}(v)$ in the logarithmic scale, and (e) the peak-to-peak amplitude of $E_{\mathrm{THz}}(t)$ and the upper cut-off frequency of $E_{\mathrm{THz}}(v)$. (f) THz wave generation by 0.22 -mm-thick HMQ-TMS crystals as a function of pump average power $P_{\text {pump }}(D=3.0 \mathrm{~mm})$ : the peakto-peak amplitude $E_{\mathrm{THz}}(t)$ and upper cut-off frequency in $E_{\mathrm{THz}}(v)$ (see also Figure S6 in SI). 
Large-area HMQ-TMS crystals possessing parallel and extremely flat high-quality surfaces were easily produced without any sophisticated polishing and cutting processes by using the above mentioned cleaving method. The thickness of the synthesized HMQ-TMS crystals was in the range from $0.05 \mathrm{~mm}$ to several millimeters. Very thin HMQ-TMS crystals with thickness of $<0.1 \mathrm{~mm}$ have been prepared by splitting a thin film with an adhesive tape, as used for graphene synthesis $^{52}$. Such thickness controllability, enabling group velocitymatched efficient $\mathrm{THz}$ wave generation, is a very unique feature for organic crystals.

\section{Discussion}

We have investigated the determination of the optimal group velocity-matched conditions in HMQ-TMS crystals with pumping at the wavelength of $800 \mathrm{~nm}$, by using high-quality crystals with different thicknesses prepared by the easy fabrication procedure. $\mathrm{THz}$ waves were generated by optical rectification (OR) and detected by the electro-optic sampling (EOS) method using a birefringent crystal (see Figure $3 \mathrm{a}$ ). The OR experiments were performed based on a Ti:sapphire regenerative amplifier system delivering $90 \mathrm{fs}$ pulses at a repetition rate of $1 \mathrm{kHz}$. An iris diaphragm located in front of HMQ-TMS in combination with a telescope was adjusted to obtain different beam diameters and input pump powers. Behind the iris diaphragm with diameters of $D=1.0,1.7$, and $3.0 \mathrm{~mm}$, the average pump power $P_{\text {pump }}$ was measured to be 38,62 , and $158 \mathrm{~mW}$, respectively. The generated $\mathrm{THz}$ waves were subsequently focused on a 1mm-thick $<110>$ ZnTe crystal for EOS detection.

The THz waves were generated using HMQ-TMS crystals of different thicknesses. The optical pump beam was incident normal to the cleavage ac-plane of the HMQ-TMS crystal and polarized parallel to the polar axis. The time-domain $E_{\mathrm{THz}}(t)$ traces and the corresponding frequency spectra of $E_{\mathrm{THz}}(v)$ are shown in Figures $3 \mathrm{~b}, 3 \mathrm{c}$, and $3 \mathrm{~d}$. The field amplitude and the spectral bandwidth of the generated $\mathrm{THz}$ waves strongly depend on the crystal thickness. Figure $3 \mathrm{e}$ shows the peak-to-peak $\mathrm{THz}$ electric field as a function of crystal thickness. The upper cut-off frequency, defined in this work as the higher frequency limit in the spectrum at which the amplitude of $E_{\mathrm{THz}}(v)$ becomes larger than the average noise level (1.0 in arbitrary units), is shown by the horizontal dotted line in Figure $3 \mathrm{~d}$. In this way, the optimal thickness for broadband and intense $\mathrm{THz}$ wave generation in HMQ-TMS at $800 \mathrm{~nm}$ pumping was determined to be between 0.16 and $0.22 \mathrm{~mm}$, where the highest peak-to-peak $\mathrm{THz}$ electric field and the largest spectral bandwidth have also been obtained (see Figure $3 \mathrm{e}$ ). It is noted that the THz cut-off frequency obtained with the optimal thickness is mainly limited by the pulse width of the pump laser (90 fs) and the detection bandwidth of $\mathrm{ZnTe}$ used, instead of the phase matching bandwidth of HMQ-TMS crystal.

We have also examined the $\mathrm{THz}$ generation efficiency as a function of the incident pump power $P_{\text {pump. }}$. The HMQ-TMS crystal with a thickness of $0.22 \mathrm{~mm}$ has been used with a relatively large aperture $(D=3.0 \mathrm{~mm})$. The peak-to-peak amplitude of $E_{\mathrm{THz}}(t)$ and upper cut-off frequency of $E_{\mathrm{THz}}(\mathrm{v})$ are shown in Figure 3f, and additional details are shown in Figure S6 in SI. Both peak-to-peak amplitude of $E_{\mathrm{THz}}(t)$ and upper cut-off frequency of $E_{\mathrm{THz}}(\mathrm{v})$ increased with the increase in the incident power, and started to slowly saturate at $P_{\text {pump }}$ above $\sim 150 \mathrm{~mW}$. At the average pump power $P_{\text {pump }}$ of $316 \mathrm{~mW}$, we measured the peak-to-peak amplitude $E_{\mathrm{THz}}(t)=72 \mathrm{kV} / \mathrm{cm}$ and large spectral bandwidth with the upper cut-off frequency of $6.0 \mathrm{THz}$, even though larger amplitude and spectral bandwidth are expected. It is noteworthy that such saturation behavior at higher pump power is generally caused by a strongly modulated phase shift induced by the nonlinear interaction of the intense $\mathrm{THz}$ waves with the optical probe pulses in ZnTe EOS crystal and by the magnitude of its EO coefficient ${ }^{53}$. Nevertheless, the potential applicability of HMQ-TMS crystals for the high-power broadband $\mathrm{THz}$ generation has been clearly demonstrated.

In order to confirm the superior $\mathrm{THz}$ generation characteristics of HMQ-TMS crystals, benchmark inorganic ZnTe crystals and organic polyene OH1, stilbazolium DAST, and quinolinium HMQ$\mathrm{T}$ crystals were used for comparison. The results of $\mathrm{THz}$ wave generation in the 0.16-mm-thick HMQ-TMS, 0.27-mm-thick OH1, and 1.0 -mm-thick ZnTe crystals when a large beam diameter $(D=$ $3.0 \mathrm{~mm}$ ) is used, are shown in Figures $4 \mathrm{a}$ and $4 \mathrm{~b}$. The results of $\mathrm{THz}$ wave generation are summarized in Table 1 , from which one can expect that HMQ-TMS crystal exhibits the highest THz generation efficiency. The peak-to-peak amplitude $E_{\mathrm{THz}}(t)$ of $\mathrm{THz}$ waves generated in HMQ-TMS is 2.7- and 1.8-times higher than that of $\mathrm{ZnTe}$ and $\mathrm{OH} 1$ crystals, respectively. In addition, the peak-to-peak amplitude $E_{\mathrm{THz}}(t)$ is remarkably higher than that (only 0.56 -fold of that generated in 1 -mm-thick ZnTe) of the 0.5 -mm-thick DAST crystal, as reported previously under similar experimental conditions (pump wavelength: $800 \mathrm{~nm}$, pulse width: $100 \mathrm{fs}$, and $P_{\text {pump }}=$ $40 \mathrm{~mW})^{14}$. Figure $4 \mathrm{~b}$ (see also its logarithm scale in Figure S7 in SI) indicates that HMQ-TMS crystal shows higher intensity than $\mathrm{OH} 1$ and ZnTe crystals, across the entire range of the generated $\mathrm{THz}$ frequency. Furthermore, the upper cut-off frequency of HMQ-TMS (6.0 THz, see Figure S7 in SI) is significantly higher than that of $\mathrm{ZnTe}$ crystal $(3.8 \mathrm{THz})$ and slightly higher than that of $\mathrm{OH} 1$ crystal $(5.3 \mathrm{THz})$.

Figures $4 \mathrm{c}$ and $4 \mathrm{~d}$ show a comparison between HMQ-TMS and HMQ-T crystals. Here, a smaller beam diameter of $D=1.0 \mathrm{~mm}$ had to be used for comparison because of the limited size of the grown HMQ-T crystal. The THz generation efficiency of the $0.16-\mathrm{mm}$ thick HMQ-TMS is found to be comparable that of the $0.58-\mathrm{mm}$ thick HMQ-T; the peak-to-peak amplitude of $E_{\mathrm{THz}}(t)$ is $34 \mathrm{kV} / \mathrm{cm}$ for HMQ-TMS and $41 \mathrm{kV} / \mathrm{cm}$ for HMQ-T crystal. As shown in Figure $4 \mathrm{~d}$, except in the frequency range of 0.45 to $1.15 \mathrm{THz}$, the HMQ-TMS crystal shows higher THz signal amplitude up to the cutoff frequency. In addition, both HMQ-T and DAST crystals exhibit a dip in the transmission curve around $1.1 \mathrm{THz}$ due to strong absorption by the phonon mode related to the methylbenzenesulfonate anion $^{13,37}$, while in HMQ-TMS crystals, such a dip does not appear or is negligible. HMQ-TMS crystals with large sizes therefore offer strong advantages for gap-free broadband and high-power $\mathrm{THz}$ generation compared with HMQ-T crystals.

In summary, we have reported a novel highly efficient electrooptic organic HMQ-TMS crystal. This HMQ-TMS crystal fulfills all the requirements for $\mathrm{THz}$ wave generation, such as large electro-optic response and optimal crystal characteristics including large crystal size with desired facets, high environmental and thermal stabilities, high optical quality, wide transparency range, and the possibility to control the crystal thickness. HMQ-TMS crystals with different thicknesses in the range of $0.05 \mathrm{~mm}$ to several millimeters, easily controlled by employing a simple cleaving method, could be fabricated, which allowed optimization of the optical-to- $\mathrm{THz}$ frequency conversion process for pumping at a desired optical pump frequency. At the widely used pump wavelength of $800 \mathrm{~nm}$, HMQTMS crystals exhibit better crystal properties and $\mathrm{THz}$ generation characteristics, and delivered broader $\mathrm{THz}$ spectral coverage and higher $\mathrm{THz}$ generation efficiency as compared with the benchmark inorganic and organic crystals. Thus, the newly developed HMQTMS crystal offers a high potential for efficient broadband $\mathrm{THz}$ wave generation with pumping in the broad NIR wavelength range.

\section{Methods}

Single-crystal X-ray structure analysis. HMQ-TMS single crystals are grown by a rapid cooling method in a methanol solution. $\mathrm{C}_{28} \mathrm{H}_{29} \mathrm{NO}_{5} \mathrm{~S}, M_{r}=491.58$, monoclinic, space group $P n, a=11.1192(3) \AA, b=7.9773(2) \AA, c=14.4921(4) \AA, \beta=$ 106.2569(9) ${ }^{\circ}, V=1234.07(6) \AA^{3}, Z=2, T=290(1) \mathrm{K}, \mu(\mathrm{MoK} \alpha)=0.171 \mathrm{~mm}^{-1}$. Among the 11774 reflections collected in the angular range $\theta=3.19^{\circ}-27.44^{\circ}$ using a $\omega$ scan on a Rigaku R-axis Rapid S diffractometer, 5291 were unique reflections $\left(R_{\text {int }}\right.$ 
(a)

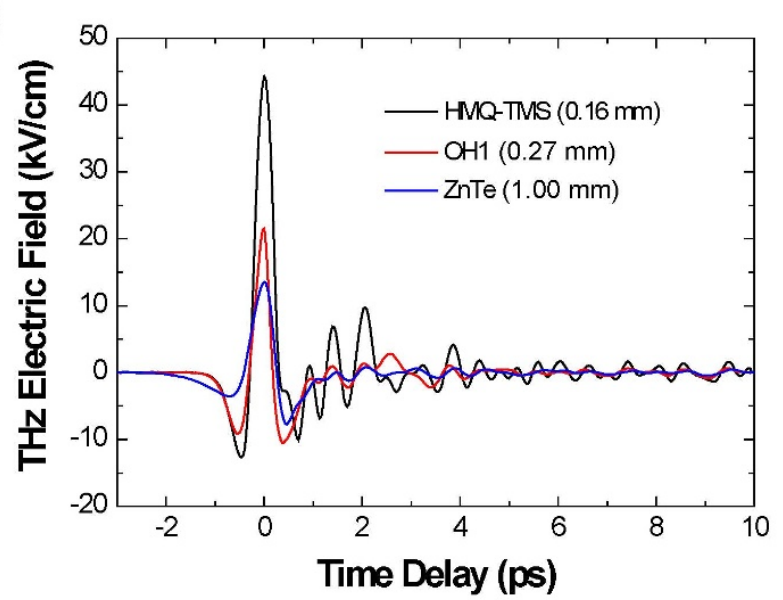

(c)

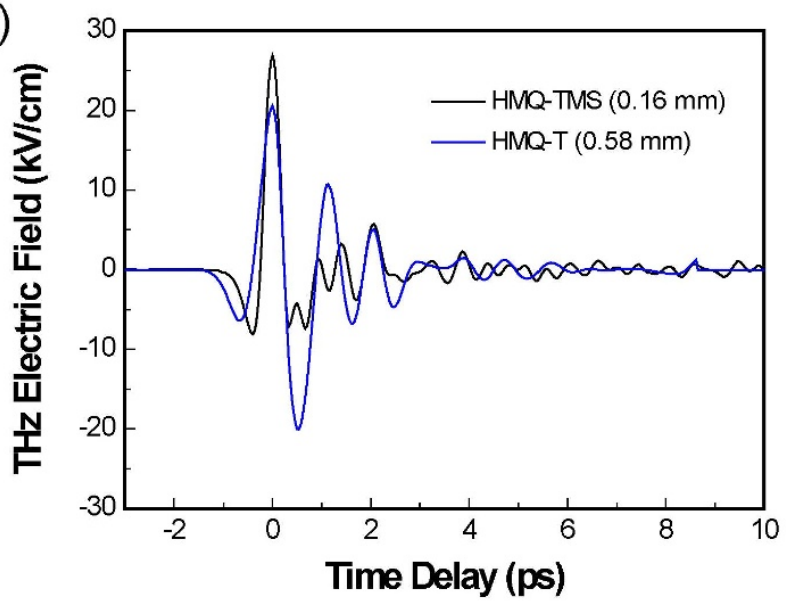

(b)

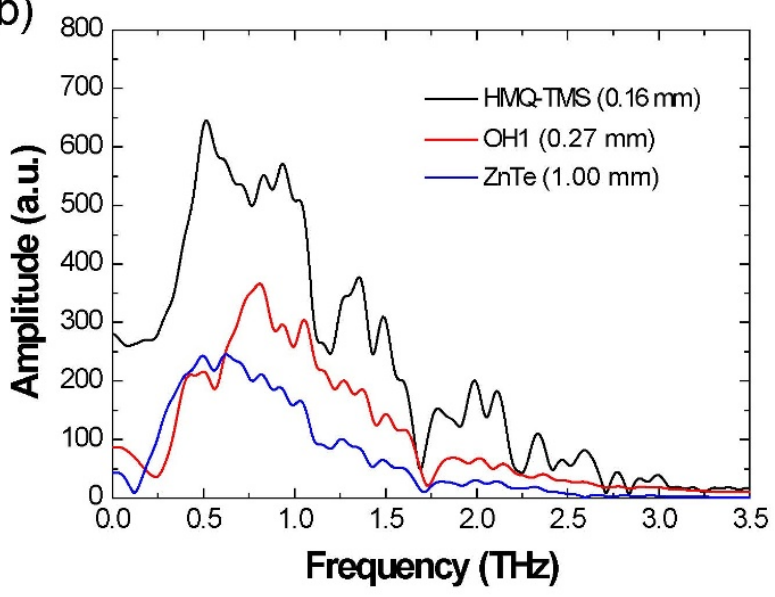

(d)

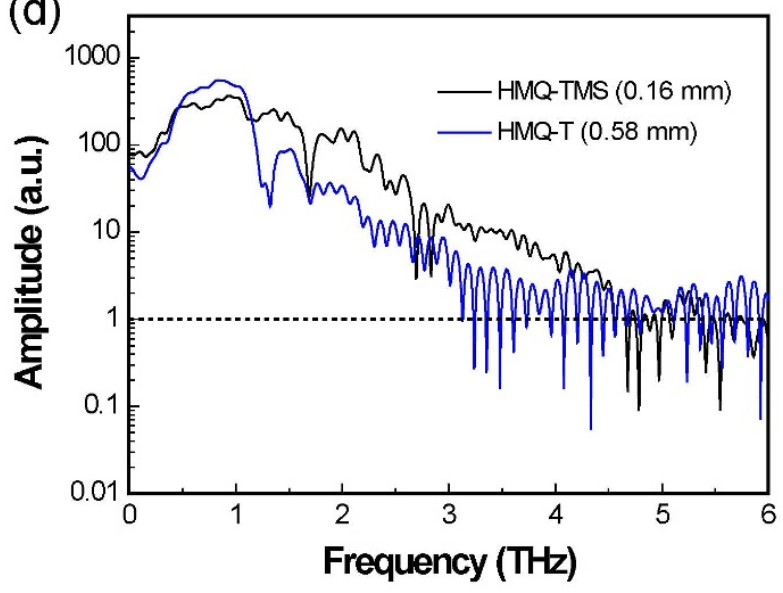

Figure $4 \mid$ High-power broadband THz wave generation. THz wave generation in 0.16-mm-thick HMQ-TMS, 0.27-mm-thick OH1, and 1.0-mm-thick ZnTe crystals $\left(P_{\text {pump }}=158 \mathrm{~mW}, D=3.0 \mathrm{~mm}\right)$ : (a) the time-domain $E_{\mathrm{THz}}(t)$ trace and $(\mathrm{b})$ the corresponding frequency spectra of $E_{\mathrm{THz}}(v)$. THz wave generation in 0.16-mm-thick HMQ-TMS crystal and 0.58-mm-thick HMQ-T crystal $\left(P_{\text {pump }}=38 \mathrm{~mW}, D=1.0 \mathrm{~mm}\right):(\mathrm{c})$ the time-domain $E_{\mathrm{THz}}(t)$ trace and (d) the corresponding frequency spectra of $E_{\mathrm{THz}}(\mathrm{v})$.

$=0.0149$, completeness $=99.5 \%)$. The structure was solved and refined against $\mathrm{F}^{2}$ using SHELX97 $7^{54}, 317$ variables, $w R_{2}=0.1129, R_{1}=0.0389\left(\mathrm{Fo}^{2}>2 \sigma\left(\mathrm{Fo}^{2}\right)\right)$, GOF $=$ 1.055 , and $\mathrm{max} / \mathrm{min}$ residual electron density $0.318 /-0.255 \mathrm{e}^{-3}$. Further details of the crystal structure investigation(s) may be obtained from the Cambridge Crystallographic Data Centre (CCDC, 12 Union Road, Cambridge CB2 1EZ (UK); tel.: (+44)1223-336-408, fax: (+44)1223-336-033, e-mail: deposit@ccdc.cam.ac.uk) on quoting the depository number CCDC-931931.

THz wave generation experiments. Our experimental setup for $\mathrm{THz}$ wave generation was based on a Ti:sapphire regenerative amplifier system (Spitfire Ace, Spectra Physics) that delivers $90 \mathrm{fs}$ pulses with a center wavelength of $800 \mathrm{~nm}$ at a repetition rate of $1 \mathrm{kHz}$. In the schematic layout shown in Figure 3a, THz waves were generated by optical rectification (OR) using femtosecond laser pulses and detected by electro-optic sampling (EOS). In order to compare the efficiency of various nonlinear optical crystals with different aperture sizes on an unbiased basis, an iris diaphragm mounted in front of the crystals was used after collimating the pump beam by using a telescope. Since frequency conversion process in a nonlinear organic crystal is quite sensitive to its orientation and tilting angle along the propagation direction, the sample mounting stage has to possess several degrees of freedom for fine tuning. The generated $\mathrm{THz}$ waves propagating through a $\mathrm{THz}$ lens pair were then focused on a 1-mm-thick $<110>\mathrm{ZnTe}$ crystal for the EOS detection. When a $\mathrm{THz}$ transient propagates in such an EO crystal, the refractive index change that is proportional to the propagating $\mathrm{THz}$ electric field is induced. A small portion of the linearly polarized $800-\mathrm{nm}$ femtosecond pulses from the laser output serves as probe and experiences the $\mathrm{THz}$ wave-induced birefringence inside the crystal. The induced polarization rotation of the probe beam is separated by employing a quarter wave plate and a Wollaston prism. The amplitude modulation of NIR probe beam is detected by two Si detectors in conjunction with a lock-in amplifier. The time-trace of the $\mathrm{THz}$ electric field is recorded as a function of time delay by moving the motorized delay stage that is mounted in the excitation beam line. The measured $\mathrm{THz}$ signal $\Delta I / I$ was then calibrated to electric field strength of $\mathrm{THz}$ waves using the relation between the signal modulation and the electro-optic coefficient of $\mathrm{ZnTe}$ crystal.

1. Tonouchi, M. Cutting-edge terahertz technology. Nat. Photonics 1, 97-105 (2007).

2. Ferguson, B. \& Zhang, X. C. Materials for terahertz science and technology. Nat. Mater. 1, 26-33 (2002).

3. Lee, Y.-S. Principles of Terahertz Science and Technology (Springer, University Press, 2010).

4. Walther, M. et al. Chemical sensing and imaging with pulsed terahertz radiation. Anal. Bioanal. Chem. 397, 1009-1017 (2010).

5. Plusquellic, D. F., Siegrist, K., Heilweil, E. J. \& Esenturk, O. Applications of terahertz spectroscopy in bio-systems. ChemPhysChem 8, 2412-2431 (2007).

6. Sirtori, C. Applied physics: bridge for the terahertz gap. Nature 417, 132-133 (2002).

7. Dragoman, D. \& Dragoman, M. Terahertz fields and applications. Progress in Quantum Electronics 28, 1-66 (2004).

8. Zheng, X., McLaughlin, C. V., Cunningham, P. \& Hayden, L. M. Organic broadband terahertz sources and sensors. J. Nanoelect. Optoelect. 2, 58-76 (2007).

9. Tani, M., Matsuura, S., Sakai, K. \& Nakashima, S.-I. Emission characteristics of photoconductive antennas based on low-temperature-grown GaAs and semiinsulating GaAs. Appl. Opt. 36, 7853-7859 (1997).

10. Xu, L., Zhang, X.-C. \& Auston, D. H. Terahertz beam generation by femtosecond optical pulses in electro-optic materials. Appl. Phys. Lett. 61, 1784-1786 (1992). 
11. Nahata, A., Weling, A. S. \& Heinz, T. F. A wideband coherent terahertz spectroscopy system using optical rectification and electro-optic sampling. Appl. Phys. Lett. 69, 2321-2323 (1996).

12. Han, P. Y., Tani, M., Pan, F. \& Zhang, X.-C. Use of the organic crystal DAST for terahertz beam applications. Opt. Lett. 25, 675-677 (2000).

13. Stillhart, M., Schneider, A. \& Günter, P. Optical properties of 4-N,Ndimethylamino- $4^{\prime}$ - $\mathrm{N}^{\prime}$-methyl-stilbazolium 2,4,6-trimethylbenzenesulfonate crystals at terahertz frequencies. J. Opt. Soc. Am. B. 25, 1914-1919 (2008).

14. Hashimoto, H., Takahashi, H., Yamada, T., Kuroyanagi, K. \& Kobayashi, T. Characteristics of the terahertz radiation from single crystals of N-substituted 2methyl-4-nitroaniline. J. Phys.: Condens. Matter. 13, L529-L537 (2001).

15. Brunner, F. D. et al. A hydrogen-bonded organic nonlinear optical crystal for high-efficiency terahertz generation and detection. Opt. Express 16, 16496-16508 (2008).

16. Stepanov, A. G., Hebling, J. \& Kuhl, J. Efficient generation of subpicosecond terahertz radiation by phase-matched optical rectification using ultrashort laser pulses with tilted pulse fronts. Appl. Phys. Lett. 15, 3000-3002 (2003).

17. Hirori, H., Doi, A., Blanchard, F. \& Tanaka, K. Single-cycle terahertz pulses with amplitudes exceeding $1 \mathrm{MV} / \mathrm{cm}$ generated by optical rectification in $\mathrm{LiNbO}_{3}$. Appl. Phys. Lett. 98, 091106 (2011).

18. Xie, X., Dai, J. \& Zhang, X.-C. Coherent control of THz wave generation in ambient air. Phys. Rev. Lett. 96, 075005 (2006).

19. Dai, J., Liu, J. \& Zhang, X.-C. Terahertz wave air photonics: Terahertz wave generation and detection with laser-induced gas plasma. IEEE J. Sel. Top. Quantum Electron. 17, 183-190 (2011).

20. Ruchert, C., Vicario, C. \& Hauri, C. P. Scaling submillimeter single-cycle transients toward megavolts per centimeter field strength via optical rectification in the organic crystal OH1. Opt. Lett. 37, 899-901 (2012).

21. Uchida, H. et al. Widely tunable broadband terahertz radiation generation using a configurationally locked polyene 2-[3-(4-hydroxystyryl)-5,5-dimethylcyclohex2 -enylidene] malononitrile crystal via difference frequency generation. Appl. Phys. B. 111, 489-493 (2013).

22. Suizu, K., Miyamoto, K., Yamashita, T. \& Ito, H. High-power terahertz-wave generation using DAST crystal and detection using mid-infrared power meter Opt. Lett. 32, 2885-2887 (2007).

23. Kwon, O-P. et al. Organic phenolic configurationally locked polyene single crystals for electro-optic and terahertz wave applications. Adv. Funct. Mater. 18, 3242-3250 (2008).

24. Choi, E.-Y., Seo, J.-Y., Jazbinsek, M. \& Kwon, O-P. Thickness control of highly efficient organic electro-optic phenolic polyene crystals by metal acetates. Cryst. Growth Des. 9, 4269-4272 (2009).

25. Bosshard, Ch., Bösch, M., Liakatas, I., Jäger, M. \& Günter, P. in Nonlinear Optical Effects and Materials (Springer-Verlag, 2000).

26. Sun, S. S. \& Dalton, L. R. Introduction to Organic Electronic and Optoelectronic Materials and Devices (CRC Press, 2008).

27. Nalwa, H. S. \& Miyata, S. Nonlinear Optics of Organic and Molecules and Polymers (CRC Press, 1997)

28. Radhakrishnan, T. P. Molecular structure, symmetry, and shape as design elements in the fabrication of molecular crystals for second harmonic generation and the role of molecules-in-materials. Acc. Chem. Res. 41, 367-376 (2008).

29. Kwon, O-P. et al. Organic nonlinear optical crystals based on configurationally locked polyene for melt growth. Chem. Mater. 18, 4049-4054 (2006).

30. Kwon, O-P. et al. New organic nonlinear optical polyene crystals and their unusual phase transitions. Adv. Funct. Mater. 17, 1750-1756 (2007).

31. Coe, B. J. et al. Diquat derivatives: highly active, two-dimensional nonlinear optical chromophores with potential redox switchability. J. Am. Chem. Soc. 132, 10498-10512 (2010)

32. Marder, S. R., Perry, J. W. \& Yakymyshyn, C. P. Organic salts with large secondorder optical nonlinearities. Chem. Mater. 6, 1137-1147 (1994).

33. Pan, F. et al. Electro-optic properties of the organic salt 4-N,N-dimethylamino-4' N'-methyl-stilbazolium tosylate. Appl. Phys. Lett. 69, 13-15 (1996).

34. Yang, Z. et al. Large-size bulk and thin-film stilbazolium-salt single crystals for nonlinear optics and THz generation. Adv. Funct. Mater. 17, 2018-2023 (2007).

35. Coe, B. J. et al. Quadratic nonlinear optical properties of N-aryl stilbazolium dyes. Adv. Funct. Mater. 12, 110-116 (2002).

36. Mutter, L., Brunner, F. D., Yang, Z., Jazbinsek, M. \& Günter, P. Linear and nonlinear optical properties of the organic crystal DSTMS. J. Opt. Soc. Am. B 24, 2556-2561 (2007)

37. Kim, P.-J. et al. Highly efficient organic $\mathrm{THz}$ generator pumped at near-infrared: quinolinium single crystals. Adv. Funct. Mater. 22, 200-209 (2012).

38. Sliker, T. R. \& Jost, J. M. "Linear electro-optic effect and refractive indices of cubic ZnTe.” J. Opt. Soc. Am. 56, 130-131 (1966)
39. Kim, P.-J. et al. New acentric quinolinium crystal with high order parameter for nonlinear optical and electro-optic applications. CrystEngComm. 14, 3633-3637 (2012).

40. Chantrapromma, S., Jindawong, B. \& Fun, H.-K. 2-[(E)-2-(4-Hydroxy-3methoxyphenyl)ethenyl]-1-methylquinolinium 4-methylbenzenesulfonate. Acta Cryst. E63, o4928-o3929 (2007).

41. Chantrapromma, S., Jindawong, B., Fun, H.-K. \& Patil, P. S. Crystal and molecular structures of 2-[(E)-2-(4-Hydroxy-3-methoxyphenyl)ethenyl]-1methylquinolinium 4-methoxybenzenesulfonate. Anal. Sci. 23, x81-x82 (2007).

42. Perdew, J. P. Density-functional approximation for the correlation energy of the inhomogeneous electron gas. Phys. Rev. B 33, 8822-8824 (1986).

43. Becke, A. D. A new mixing of Hartree-Fock and local density-functional theories. J. Chem. Phys. 98, 1372-1378 (1993).

44. Kwon, S.-J. et al. Highly nonlinear optical configurationally locked triene Crystals based on 3,5-dimethyl-2-cyclohexen-1-one. J. Phys. Chem. C 112, 7846-7852 (2008).

45. Kim, P.-J. et al. Acentric nonlinear optical N-benzyl stilbazolium crystals with high environmental stability and enhanced molecular nonlinearity in solid state. CrystEngComm. 13, 444-451 (2011).

46. Sun, Z. et al. Engineering of acentric stilbazolium salts with large second-order optical nonlinearity and enhanced environmental stability. Cryst. Growth Des. 12, 6181-6187 (2012).

47. Seo, J.-Y. et al. Large-size pyrrolidine-based polyene single crystals suitable for terahertz wave generation. Cryst. Growth Des. 9, 5003-5005 (2009).

48. Brahadeeswaran, S. et al. Growth of ultrathin and highly efficient organic nonlinear optical crystal 4' -dimethylamino-N-methyl-4-stilbazolium pchlorobenzenesulfonate for enhanced terahertz efficiency at higher frequencies. Cryst. Growth Des. 13, 415-421 (2013).

49. Manetta, S., Ehrensperger, M., Bosshard, Ch. \& Günter, P. Organic thin film crystal growth for nonlinear optics: present methods and exploratory developments. C. R. Physique 3, 449-462 (2002).

50. Kwon, S.-J., Jazbinsek, M., Kwon, O-P. \& Günter, P. Crystal growth and morphology control of $\mathrm{OH} 1$ organic electrooptic crystals. Cryst. Growth Des. 10, 1552-1558 (2010).

51. Ruiz, B., Jazbinsek, M. \& Günter, P. Crystal growth of DAST. Cryst. Growth Des. 8, 4173-4184 (2008).

52. Novoselov, K. S. et al. Electric field effect in atomically thin carbon films. Science 306, 666-669 (2004).

53. Jewariya, M., Nagai, M. \& Tanaka, K. Enhancement of terahertz wave generation by cascaded $\chi^{(2)}$ processes in $\mathrm{LiNbO}_{3}$. J. Opt. Soc. Am. B 26, A101-A106 (2009). 54. Sheldrick, G. M. A short history of SHELX. Acta Crystallogr. A64, 112-122 (2008).

\section{Acknowledgments}

This work has been supported by Mid-career Researcher Program (NRF-2013R1A2A2A01007232), Priority Research Centers Program (2009-0093826) through the National Research Foundation of Korea (NRF) funded by the Ministry of Education, Science and Technology (MEST), and NRF (2011-0017494 \& 2008-0061906) funded by MEST.

\section{Author contributions}

J.-H.J. and B.-J.K. are equally contributed. Synthesis and characterization of organic crystals were performed by O-P.K., J.-H.J., J.-S.K., S.-H.L. and S.-C.L. Experiments on THz wave generation were done by F.R., B.-J.K. and I.-H.B. The crystal structure analysis was performed by H.Y. Quantum chemical calculations were done by M.J., J.K. and Y.S.L. AFM measurements were done by J.-H.L. and J.-H.K.. O-P.K. and F.R. conceived and supervised the project. All authors have discussed and reviewed the manuscript.

\section{Additional information}

Supplementary information accompanies this paper at http://www.nature.com/ scientificreports

Competing financial interests: The authors declare no competing financial interests. How to cite this article: Jeong, J.-H. et al. High-power Broadband Organic THz Generator Sci. Rep. 3, 3200; DOI:10.1038/srep03200 (2013).

This work is licensed under a Creative Commons AttributionNonCommercial-NoDerivs 3.0 Unported license. To view a copy of this license, visit http://creativecommons.org/licenses/by-nc-nd/3.0 